International Journal of Modern Physics A

(C) World Scientific Publishing Company

\title{
WHY SCREENING EFFECTS DO NOT INFLUENCE THE CASIMIR FORCE*
}

\author{
V. M. MOSTEPANENKO ${ }^{\dagger}$ \\ Institute for Theoretical Physics, Leipzig University, D-04009, Leipzig, Germany \\ Vladimir.Mostepanenko@itp.uni-leipzig.de \\ R. S. DECCA \\ Department of Physics, Indiana University-Purdue University Indianapolis, IN 46202, USA \\ E. FISCHBACH \\ Department of Physics, Purdue University, West Lafayette, IN 47907, USA \\ B. GEYER and G. L. KLIMCHITSKAYA ${ }^{\ddagger}$ \\ Institute for Theoretical Physics, Leipzig University, D-04009, Leipzig, Germany \\ D. E. KRAUSE \\ Physics Department, Wabash College, Crawfordsville, IN 47933, USA \\ D. LÓPEZ \\ Center of Nanoscale Materials, Argonne National Laboratory, Argonne, IL 60439, USA \\ U. MOHIDEEN \\ Department of Physics and Astronomy, University of California, Riverside, CA 92521, USA
}

Received 20 October 2008

Revised 22 January 2009

\begin{abstract}
The Lifshitz theory of dispersion forces leads to thermodynamic and experimental inconsistencies when the role of drifting charge carriers is included in the model of the dielectric response. Recently modified reflection coefficients were suggested that take into account screening effects and diffusion currents. We demonstrate that this theoretical approach leads to a violation of the third law of thermodynamics (Nernst's heat theorem) for a wide class of materials and is excluded by the data from two recent experiments. The physical reason for its failure is explained by the violation of thermal equilibrium, which is the fundamental applicability condition of the Lifshitz theory, in the presence of drift
\end{abstract}

*This paper is the presentation of two talks given by V. M. Mostepanenko and by B. Geyer. $\dagger$ On leave from Noncommercial Partnership "Scientific Instruments", Moscow, Russia

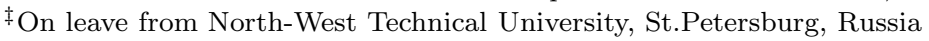


and diffusion currents.

Keywords: Casimir force; screening effects; Nernst's heat theorem.

PACS numbers: 12.20.-m, 42.50.Ct, 78.20.Ci

\section{Introduction}

In the last few years the Casimir effect has attracted increasing attention due to prospective applications in both fundamental physics and nanotechnology. Sixty years ago H. B. G, Casimir 1 made his famous discovery that two electrically neutral parallel ideal metal plates spaced at some separation $a$ in vacuum attract each other. Casimir explained this effect as due to the alterations in the spectrum of zero-point oscillations of the electromagnetic field introduced by the presence of the plates. In the simplest approach, the Casimir effect can be described theoretically using quantum field theory with boundary conditions. The case of real material plates was considered by Lifshitz, $\frac{2}{2}$ who described material properties by means of a frequency-dependent dielectric permittivity. The Lifshitz theory was successfully applied to the interpretation of measurement data in several experiments measuring the Casimir force. $\underline{3}$

Further investigations revealed that the Lifshitz theory at nonzero temperature leads to problems when the relaxation of free charge carriers is included in the model of the dielectric response. This was shown to lead to both thermodynamically and experimental inconsistencies $\underline{4}-\underline{9}$ Problems arise in the zero-frequency contribution of the Lifshitz formula when the free charge carriers are described by the dielectric permittivity of the Drude model. The Lifshitz theory was found to be thermodynamically consistent and in agreement with the experimental data if the free electrons in metals are described by the dielectric permittivity of the plasma model $10-12$ For dielectric and semiconductor materials, consistency with thermodynamics and experiment is achieved if the dc conductivity is neglected or charge carriers are described by means of the plasma model depending on whether the concentration of charge carriers is below or above the critical value, respectively 13

Recently, an alternative approach to the description of free charge carriers in the Lifshitz theory was suggested $14-16$ which takes into account screening effects and diffusion currents. Within this approach, the macroscopic characteristic of the plate material (the dielectric permittivity) is supplemented with a microscopic quantity (the density of free charge carriers). Below we consider the most typical configuration of two thick parallel plates (semispaces) and demonstrate that this approach is thermodynamically and experimentally inconsistent. The reason for this failure is the violation of thermal equilibrium which is the basic applicability condition of the Lifshitz theory.

The structure of the paper is as follows. In Sec. 2 we briefly formulate problems which arise when the Lifshitz theory is applied to materials with nonzero conductivity. Section 3 contains the formulation of approaches taking into account the 
charge screening of free carriers. In Sec. 4 it is demonstrated that these approaches are in conflict with thermodynamics. Section 5 is devoted to the comparison of theoretical results obtained with the inclusion of the screening effects and available experimental data. In Sec. 6 the reader will find our conclusions and discussion.

\section{Problems of the Lifshitz Theory in Application to Materials with Nonzero Conductivity}

At nonzero temperature all materials have a nonzero conductivity. For metals and metallic-type semiconductors this conductivity can be rather large, and does not go to zero when the temperature vanishes. For dielectrics and some semiconductors (intrinsic ones and those with dopant concentration below critical) conductivity is much smaller than for metals, and goes to zero together with temperature. In the Lifshitz theory, the free energy per unit area in the configuration of two semispaces described by the dielectric permittivity $\varepsilon(\omega)$ is given by

$$
\begin{gathered}
\mathcal{F}(a, T)=\frac{k_{B} T}{2 \pi} \sum_{l=0}^{\infty} \int_{0}^{\infty} k_{\perp} d k_{\perp}\left\{\ln \left[1-r_{\mathrm{TM}}^{2}\left(\mathrm{i} \xi_{l}, k_{\perp}\right) \mathrm{e}^{-2 a q_{l}}\right]\right. \\
\left.+\ln \left[1-r_{\mathrm{TE}}^{2}\left(\mathrm{i} \xi_{l}, k_{\perp}\right) \mathrm{e}^{-2 a q_{l}}\right]\right\}
\end{gathered}
$$

Here, $a$ is the separation distance between the semispaces, $k_{\perp}$ is the magnitude of the wave vector in the plane of boundary plates, the primed sum adds a multiple $1 / 2$ to the term with $l=0, \xi_{l}=2 \pi k_{B} T l / \hbar$ with $l=0,1,2, \ldots$ are the Matsubara frequencies, and $k_{B}$ is the Boltzmann constant. Equation (11) is derived under the condition that the plates (semispaces) are at a temperature $T$ in thermal equilibrium with the environment. The reflection coefficients for two independent polarizations of the electromagnetic field coincide with the Fresnel ones calculated along the imaginary frequency axis,

$$
r_{\mathrm{TM}}\left(\mathrm{i} \xi_{l}, k_{\perp}\right)=\frac{\varepsilon_{l} q_{l}-k_{l}}{\varepsilon_{l} q_{l}+k_{l}}, \quad r_{\mathrm{TE}}\left(\mathrm{i} \xi_{l}, k_{\perp}\right)=\frac{q_{l}-k_{l}}{q_{l}+k_{l}},
$$

where

$$
q_{l}^{2}=k_{\perp}^{2}+\frac{\xi_{l}^{2}}{c^{2}}, \quad k_{l}^{2}=k_{\perp}^{2}+\varepsilon_{l} \frac{\xi_{l}^{2}}{c^{2}}, \quad \varepsilon_{l} \equiv \varepsilon\left(\mathrm{i} \xi_{l}\right) .
$$

Originally, Eqs. (1)-(3) were mostly applied to dielectrics with the dc conductivity neglected. For such materials the dielectric permittivity can be written in the form 17

$$
\varepsilon(\mathrm{i} \xi)=1+\sum_{j=1}^{K} \frac{f_{j}}{\omega_{j}^{2}+\xi^{2}+\gamma_{j} \xi},
$$

where $\omega_{j} \neq 0$ are the oscillator frequencies, $f_{j}$ are the oscillator strengths and $\gamma_{j}$ are the relaxation parameters. This equation describes the dielectric response of core electrons. The free energy (10) with the dielectric permittivity (4) is in perfect 
agreement with thermodynamics. Specifically, the Casimir entropy vanishes with temperature. 13

Problems with thermodynamics arise when the conductivity $\sigma$ of the plate material is taken into account. For metals and metallic-type semiconductors the dielectric permittivity can be modelled by means of the Drude model,

$$
\tilde{\varepsilon}(\mathrm{i} \xi)=\varepsilon(\mathrm{i} \xi)+\frac{4 \pi \sigma(\mathrm{i} \xi)}{\xi}=\varepsilon(\mathrm{i} \xi)+\frac{\omega_{p}^{2}}{\xi(\xi+\gamma)},
$$

where $\omega_{p}$ is the plasma frequency and $\gamma$ is the relaxation parameter of free electrons. Simple expressions for the quantities entering Eq. (5) are! 18

$$
\sigma(\mathrm{i} \xi)=\frac{\sigma(0)}{1+\frac{\xi}{\gamma}}, \quad \omega_{p}^{2}=\frac{4 \pi e^{2} n}{m}, \quad \sigma(0)=\mu|e| n
$$

where $\sigma(0)$ is the dc conductivity, $e$ and $m$ are the charge and effective mass of the electron, $n$ is the density of charge carriers and $\mu$ is their mobility. The substitution of the dielectric permittivity (5) into Eqs. (1)-(3) results in a negative Casimir entropy at $T=0 \mathrm{~K}$ depending on $a$ for metals with perfect crystal lattices, $\frac{4}{4}$ which is in violation of the third law of thermodynamics (the Nernst heat theorem). Recently it was claimed 19 that Ref. 4 is wrong because it uses the theory of the normal skin effect for metals with perfect crystal lattices at $T \rightarrow 0$, while in this situation one must use the theory of the anomalous skin effect. This objection is, however, incorrect. With the decrease of $T$ the application region of the normal skin effect does become narrower and the application region of the anomalous skin effect widens. However, at any $T>0$, there exists a frequency region near zero frequency, where the normal skin effect is applicable. Keeping in mind that the violation of the Nernst heat theorem originates entirely from the zero-frequency term of the Lifshitz formula, one concludes that the permittivity of the normal skin effect is appropriate for the evaluation of this term at low $T$ when considering the thermodynamic consistency of the Lifshitz theory. There is a suggestion 20121 to satisfy the Nernst heat theorem by the inclusion of impurities, but this does not solve the problem $22 \mid 23$ because according to quantum statistical physics for perfect crystal lattices the Casimir entropy at zero temperature must be equal to zero.

Another problem is the experimental inconsistency of the Lifshitz theory combined with the dielectric permittivity (5). This was demonstrated in a series of three successive experiments on the dynamic determination of the Casimir pressure between two gold plates. $6 / 24$ For example, in Fig. 1 we demonstrate the comparison between the measurement data of the most precise third experiment $7 / 8$ and theoretical Casimir pressures computed using the Drude model (5) and the generalized plasma-like model of Refs. 10 12 [dielectric permittivity (5) with $\gamma=0$ ]. In Fig. 1(a) the Casimir pressures computed using the Drude model approach and the generalized plasma-like model are shown as dark-gray and light-gray bands, respectively. The experimental data are shown as crosses. The widths of the bands and the sizes of the arms of the crosses are determined at a $95 \%$ confidence level. In Fig. 1(b), the 

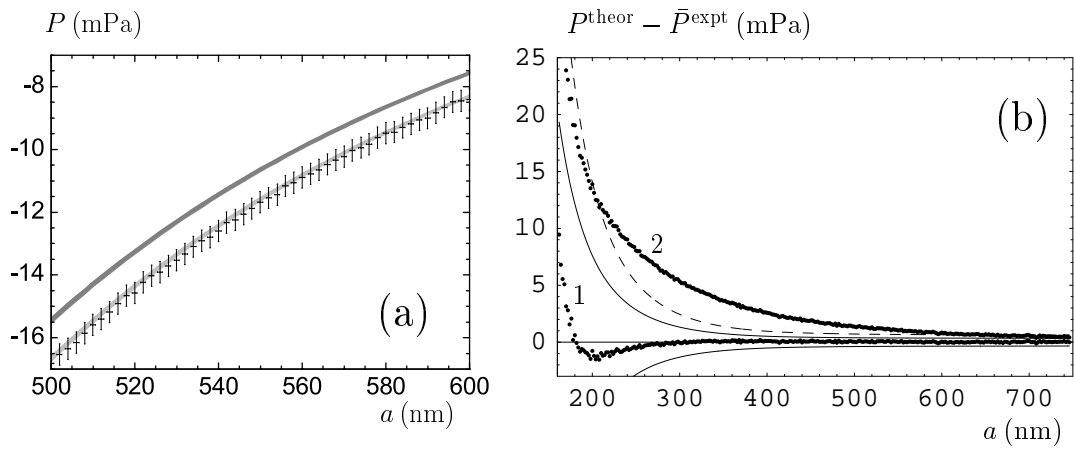

Fig. 1. (a) The crosses show the measured mean Casimir pressures together with the absolute errors in the separation and pressure as a function of the separation. The theoretical Casimir pressures computed using the generalized plasma-like model and the optical data extrapolated by the Drude model are shown by the light-gray and dark-gray bands, respectively. (b) The differences of the theoretical and the mean experimental Casimir pressures between two Au plates versus separation are shown as dots. The theoretical results are calculated using the Lifshitz theory at room temparature using the generalized plasma-like model (the dots labeled 1) and the Drude model approach (the dots labeled 2). The solid and dashed lines indicate the boundaries of $95 \%$ and $99.9 \%$ confidence intervals, respectively.

differences of the theoretical Casimir pressures computed using Eq. (5), $P_{D}^{\text {theor }}(a)$, and the mean experimental Casimir pressures, $\bar{P}^{\operatorname{expt}}(a)$, are shown as dots labeled 2. The differences of the theoretical pressures computed using the generalized plasmalike permittivity [Eq. (5) with $\gamma=0$ ], $P_{g p}^{\text {theor }}(a)$, and $\bar{P}^{\operatorname{expt}}(a)$ are shown as dots labeled 1. The solid and dashed lines indicate the boundaries of $95 \%$ and $99.9 \%$ confidence intervals, respectively. As can be seen in Fig. 1(a,b), the generalized plasma-like model is consistent with data, whereas the Drude permittivity taking into account the relaxation properties connected with a drift current of conduction electrons is experimentally excluded.
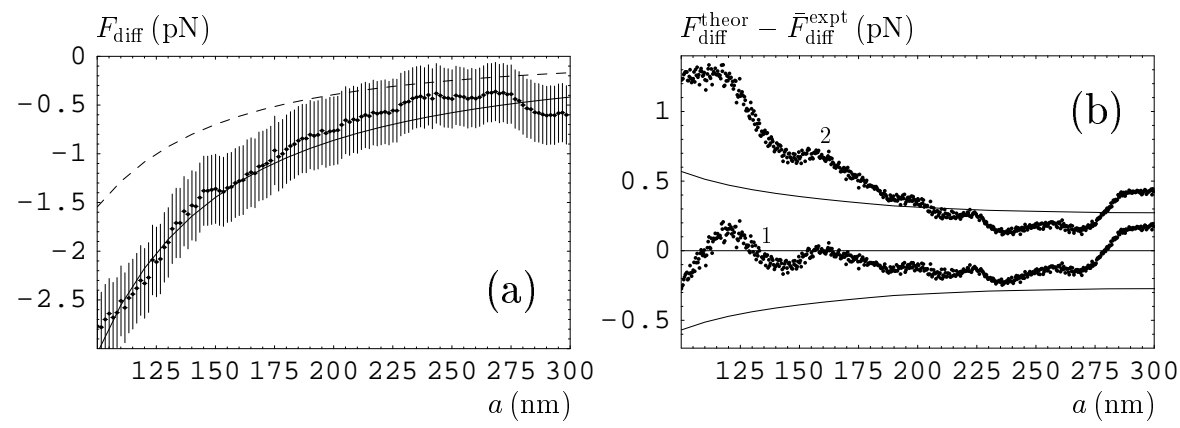

Fig. 2. (a) The measured force differences are shown as crosses versus separation $a$. The solid and dashed lines present the theoretical results calculated with the neglected and included dc conductivity of high-resistivity $\mathrm{Si}$ in the dark phase, respectively. (b) Theoretical minus mean experimental differences of the Casimir force for the Lifshitz theory with neglected (label 1) and included (label 2) dc conductivity of high-resistivity Si in the dark phase. The solid lines indicate $95 \%$ confidence intervals. 
Now we consider dielectric plates, whose conductivity vanishes with $T$, and take into account the small dc conductivity using the first equality in Eq. (5) in the calculation of the Casimir free energy and entropy [the second equality in Eq. (5) is related only to metals]. In this case the Casimir entropy at $T=0$ takes a positive value depending on $a \stackrel{5|13| 25 \mid 26}{,}$ i.e., the Nernst heat theorem is violated. Theoretical results computed with inclusion of the dc conductivity of the dielectric are in disagreement with the experimental results on the measurement of the Casimir force between a gold sphere and a silicon plate illuminated with laser pulses. $27 / 28$ As an illustration, in Fig. 2(a) the mean measured differences of the Casimir forces in the presence and in the absence of light on the plate (the absorbed power is equal to $4.7 \mathrm{~mW}$ ), $\bar{F}_{\text {diff }}^{\text {expt }}$, are shown as crosses (the arm sizes are determined at a $95 \%$ confidence level). The theoretical differences, $F_{\text {diff }}^{\text {theor }}$, shown by the solid line, are computed by using the dielectric permittivity of the generalized plasma-like model in the presence of light and neglecting the dc conductivity in the absence of light on the plate. The theoretical differences shown by the dashed line are computed by using the dielectric permittivity (5) with the respective values of $\sigma$ in the presence, and in the absence, of light, i.e., with included dc conductivity of the silicon plate also in the dark phase. As is seen in Fig. 2(a), the theory taking into account the conductivity of $\mathrm{Si}$ in the dark phase using Eq. (5) is experimentally excluded, whereas the theory neglecting the dc conductivity of dielectric silicon is consistent with the data.

The comparison of experiment with different theories can also be done in another manner. In Fig. 2(b) we plot as dots the theoretical minus mean experimental differences of the Casimir forces, $F_{\text {diff }}^{\text {theor }}-\bar{F}_{\text {diff }}^{\text {exp }}$, in the presence and in the absence of light. For the dots labeled 2 the values of $F_{\text {diff }}^{\text {theor }}$ are computed with the dc conductivity of Si included in the dark phase, and for dots labeled 1 the dc conductivity is neglected. The solid lines indicate the boundaries of $95 \%$ confidence intervals. As is seen in Fig. 2(b), the theory including the dc conductivity in the dark phase is excluded by the data over a wide separation region, whereas the theory neglecting the dc conductivity is experimentally consistent.

The physical reasons why the use of the dielectric permittivity (5) in the Lifshitz theory leads to problems with thermodynamics and experiment are discussed in Ref. 29. The point is that this permittivity describes the influence of the drift current of conduction electrons, an irreversible process. It is accompanied by Joule losses and the heating of the Casimir plates. To keep the temperature constant, one should allow the existence of an unidirectional flux of heat from the plates to the heat reservoir .30 This is a situation out of thermal equilibrium .31 Thus, the dielectric permittivity (5) describes processes violating thermal equilibrium which is the basic applicability condition of the Lifshitz theory. It is thus not surprising that in combination with the permittivity (5)), the Lifshitz theory becomes thermodynamicly and experimentally inconsistent. 


\section{Attempts to Generalize the Lifshitz Theory Through the Inclusion of Screening Effects}

As discussed in Sec. 2, to avoid problems in the application of the Lifshitz theory to real materials one should neglect the dc conductivity of dielectrics and describe charge carriers in metals using the generalized plasma-like dielectric permittivity. Another approach to the resolution of problems arising in the Lifshitz theory when it is applied to materials with nonzero conductivity is by including the effect of Coulomb screening by mobile charges. As discussed above, at nonzero temperature all materials contain charge carriers which can move through the medium. In the presence of mobile charge carriers the standard Coulomb potential of some external charge is replaced with the so-called screened Coulomb potential having a Yukawatype dependence on separation. ${ }^{32}$ The effect of screening leads to penetration of a static electric field into materials with nonzero conductivity to a depth of the screening length which is very small for good metals (of order the interatomic distance), but can be much larger for semiconductors.

The response of charge carriers to a spatially variable, sinusoidally varying, external electric field taking into account scattering can be described by Boltzmann transport equation. This approach was recently used 15 to describe the interaction of the fluctuating electromagnetic field with mobile charge carriers in a semiconductor plate and to find the modified reflection coefficients for the TM and TE modes. The resulting coefficients were substituted into the standard Lifshitz formula (1). Here, we consider the applicability of this approach to different materials leaving the problems of its consistency with thermodynamics and experiment for the following sections.

The Boltzmann transport equation is used for the description of irreversible nonequilibrium processes, and is not symmetric with respect to time reversal. As a result, it describes processes which lead to an increase of entropy ${ }^{33}$ In the case under consideration this equation includes both the drift current of charge carriers $\boldsymbol{j}$ and the diffusion current $e D \nabla n$, where $D$ is the diffusion coefficient. As a result, the modified TM reflection coefficient takes the form 15

$$
r_{\mathrm{TM}}^{\bmod }\left(\mathrm{i} \xi_{l}, k_{\perp}\right)=\frac{\tilde{\varepsilon}_{l} q_{l}-k_{l}-k_{\perp}^{2} \eta_{l}^{-1} \varepsilon_{l}^{-1}\left(\tilde{\varepsilon}_{l}-\varepsilon_{l}\right)}{\tilde{\varepsilon}_{l} q_{l}+k_{l}+k_{\perp}^{2} \eta_{l}^{-1} \varepsilon_{l}^{-1}\left(\tilde{\varepsilon}_{l}-\varepsilon_{l}\right)} .
$$

Here, $\varepsilon_{l}$ and $q_{l}$ are defined in Eq. (3), $\tilde{\varepsilon}_{l} \equiv \tilde{\varepsilon}\left(\mathrm{i} \xi_{l}\right)$ is defined in Eq. (5) , $k_{l}$ is given by Eq. (3) where $\varepsilon_{l}$ is replaced with $\tilde{\varepsilon}_{l}$ and

$$
\eta_{l} \equiv \eta\left(\mathrm{i} \xi_{l}\right)=\left[k_{\perp}^{2}+\kappa^{2} \frac{\varepsilon_{0}}{\varepsilon_{l}} \frac{\tilde{\varepsilon}_{l}}{\tilde{\varepsilon}_{l}-\varepsilon_{l}}\right]^{1 / 2}, \quad \varepsilon_{0} \equiv \varepsilon(0) .
$$

The screening length $1 / \kappa$ in Eq. (8) is different for different types of statistics of charge carriers (see below). The modified TE reflection coefficient, $r_{\mathrm{TE}}^{\bmod }\left(\mathrm{i} \xi_{l}, k_{\perp}\right)$, is given by the standard expressions (2) and (3), where the permittivity $\varepsilon_{l}$ is replaced by $\tilde{\varepsilon}_{l}$. 
It has been shown 34 that the problems of the standard Lifshitz theory discussed in Sec. 2 are mathematically connected with the discontinuity of the zero-frequency term of the Lifshitz formula with respect to frequency and temperature at the point $(0,0)$. The modified reflection coefficients at zero frequency take the form

$$
r_{\mathrm{TM}}^{\bmod }\left(0, k_{\perp}\right)=\frac{\varepsilon_{0} \sqrt{k_{\perp}^{2}+\kappa^{2}}-k_{\perp}}{\varepsilon_{0} \sqrt{k_{\perp}^{2}+\kappa^{2}}+k_{\perp}}, \quad r_{\mathrm{TE}}^{\bmod }\left(0, k_{\perp}\right)=0 .
$$

Note that the TM coefficient in (9) was first obtained by Pitaevski1 14 within the theoretical approach taking into account the penetration of the static component of the fluctuating field in the material of a wall in the atom-wall (Casimir-Polder) interaction. According to (9), the modified TE reflection coefficient at zero frequency takes the same value as in the Drude model approach. Mathematically, this suggests, that for metals the theoretical approach taking into account screening effects should face the same difficulties as the Drude model approach (see Sec. 2).

The reflection coefficients $r_{\mathrm{TM}, \mathrm{TE}}^{\mathrm{mod}}\left(0, k_{\perp}\right)$ can be obtained $14 \mid 29$ as the standard Fresnel reflection coefficients for an uniaxial crystal,

$$
r_{\mathrm{TM}}^{u}\left(\mathrm{i} \xi_{l}, k_{\perp}\right)=\frac{\sqrt{\varepsilon_{x l} \varepsilon_{z l}} q_{l}-k_{z l}}{\sqrt{\varepsilon_{x l} \varepsilon_{z l}} q_{l}+k_{z l}}, \quad r_{\mathrm{TE}}^{u}\left(\mathrm{i} \xi_{l}, k_{\perp}\right)=\frac{q_{l}-k_{x l}}{q_{l}+k_{x l}},
$$

where

$$
k_{x l}^{2}=k_{\perp}^{2}+\varepsilon_{x l} \frac{\xi_{l}^{2}}{c^{2}}, \quad k_{z l}^{2}=k_{\perp}^{2}+\varepsilon_{z l} \frac{\xi_{l}^{2}}{c^{2}}, \quad \varepsilon_{x l}=\varepsilon_{x}\left(\mathrm{i} \xi_{l}\right), \quad \varepsilon_{z l}=\varepsilon_{z}\left(\mathrm{i} \xi_{l}\right),
$$

if one introduces the dielectric permittivity depending on the wave vector $k_{\perp}$,

$$
\varepsilon_{x 0} \equiv \varepsilon_{x}(0)=\varepsilon_{0}, \quad \varepsilon_{z 0} \equiv \varepsilon_{z}(0)=\varepsilon_{0}\left(1+\frac{\kappa^{2}}{k_{\perp}^{2}}\right) .
$$

This means that the standard Lifshitz formula (at least its zero-frequency term) is applied to spatially dispersive materials. Such an application is controversial and has been debated in the literature ${ }^{[35]}$ The modified reflection coefficients $r_{\mathrm{TM}, \mathrm{TE}}^{\mathrm{mod}}\left(\mathrm{i} \xi_{l}, k_{\perp}\right)$ at any frequency are also claimed to be obtainable in terms of spatially nonlocal dielectric functions $\frac{15]}{15}$ is easily seen, however, that there are no such dielectric functions $\varepsilon_{x l}$ and $\varepsilon_{z l}$ which transform the reflection coefficients (10) for an uniaxial crystal into $r_{\mathrm{TM}, \mathrm{TE}}^{\mathrm{mod}}\left(\mathrm{i} \xi_{l}, k_{\perp}\right)$. To prove this, we first require that $r_{\mathrm{TE}}^{u}\left(\mathrm{i} \xi_{l}, k_{\perp}\right)$ be equal to $r_{\mathrm{TE}}^{\bmod }\left(\mathrm{i} \xi_{l}, k_{\perp}\right)$. For this purpose one must put $\varepsilon_{x l} \equiv \tilde{\varepsilon}_{l}$. Then, keeping in mind that $\tilde{\varepsilon}_{l}$ goes to infinity when $\xi \rightarrow 0$, one finds that in this limiting case $r_{\mathrm{TM}}^{u}\left(\mathrm{i} \xi_{l}, k_{\perp}\right)$ in Eq. (10) goes to unity independent of the functional form of $\varepsilon_{z l}$. Thus, there is no such function $\varepsilon_{z l}$ that would lead to the required equality $r_{\mathrm{TM}}^{u}\left(0, k_{\perp}\right)=r_{\mathrm{TM}}^{\bmod }\left(0, k_{\perp}\right)$, as defined in Eq. (9).

In Ref. 36 the modified reflection coefficients $r_{\mathrm{TM}, \mathrm{TE}}^{\bmod }\left(\mathrm{i}_{l}, k_{\perp}\right)$ at any frequency are expressed in terms of two dielectric functions $\varepsilon_{x}\left(\mathrm{i} \xi_{l}, k_{\perp}\right)$ and $\varepsilon_{z}\left(\mathrm{i} \xi_{l}, k_{\perp}\right)$ defined in the random phase approximation. 37 The obtained expression for $\varepsilon_{z}\left(\mathrm{i} \xi_{l}, k_{\perp}\right)$ is, however, incorrect. It does not transform into the dielectric permittivity of dielectric material $\varepsilon\left(\mathrm{i} \xi_{l}\right)$ defined in Eq. (4) in the limiting case $n \rightarrow 0$. This is caused by several 
mistakes in sign made in Ref. 36 and by the disagreement of phase multiples in the TM reflection coefficients used by the authors of Ref. 36 and in the method of random phase approximation. After correcting these errors one obtains

$$
\begin{aligned}
\varepsilon_{z}\left(\mathrm{i} \xi_{l}, k_{\perp}\right)=k_{\perp} & {\left[\frac{k_{l} \varepsilon_{l}+k_{\perp}^{2} \eta_{l}^{-1}\left(\tilde{\varepsilon}_{l}-\varepsilon_{l}\right)}{\varepsilon_{l} \tilde{\varepsilon}_{l}}+k_{\perp}-q_{l}-\frac{\xi_{l}^{2}}{c^{2}}\left(\frac{1}{k_{l}}-\frac{1}{q_{l}}\right)\right.} \\
& \left.+k_{\perp} \frac{\xi_{l}^{2}}{c^{2}}\left(\frac{1}{k_{\perp} k_{l}+k_{l}^{2}}-\frac{1}{k_{\perp} q_{l}+q_{l}^{2}}\right)\right]^{-1} \cdot
\end{aligned}
$$

Here, $k_{l}$ is defined by Eq. (3) where $\varepsilon_{l}$ is replaced with $\varepsilon_{x}\left(\mathrm{i} \xi_{l}, k_{\perp}\right)$. The latter coincides with $\tilde{\varepsilon}\left(\mathrm{i} \xi_{l}\right)$ from Eq. (5), i.e., it does not depend on $k_{\perp}$. It is easily seen that in the limiting case $n \rightarrow 0$ the permittivity (13) transforms into $\varepsilon_{l}=\varepsilon\left(\mathrm{i} \xi_{l}\right)$.

We emphasize that the coefficient $r_{\mathrm{TM}}^{\bmod }\left(\mathrm{i} \xi_{l}, k_{\perp}\right)$ obtained in the random phase approximation does not coincide with the coefficient $r_{\mathrm{TM}}^{u}\left(\mathrm{i} \xi_{l}, k_{\perp}\right)$ for an uniaxial crystal, as given by Eq. (10). It is notable also that the representation for the reflection coefficient $r_{\mathrm{TM}}^{\bmod }\left(\mathrm{i} \xi_{l}, k_{\perp}\right)$ by means of the dielectric permittivity $\varepsilon_{z}\left(\mathrm{i} \xi_{l}, k_{\perp}\right)$ at both zero and nonzero frequency is a phenomenological one. The point is that in the presence of a gap between the plates the translational invariance in $z$-direction, i.e., perpendicular to the plates, is violated and $\varepsilon_{z}\left(\mathrm{i} \xi_{l}, k_{\perp}\right)$ is an ill-defined quantity.

Phenomenological reflection coefficients at any frequency, depending on two spatially nonlocal dielectric permittivities $\varepsilon_{x l}, \varepsilon_{z l}$ and the screening length $1 / \kappa$, were also suggested without refer to Boltzmann transport equation. 16 At zero frequency they take the same values as in Eq. (9). This approach is a crude approximation. Given the absence of translational invariance along the $z$-axis mentioned above, it is impossible to define the dielectric permittivity $\varepsilon_{z l}$ depending on the frequency and the wave vector. Additionally, specular reflection of charge carriers on the boundary planes was assumed ${ }^{16}$ However, for spatially dispersive materials the scattering of carriers is neither specular nor diffuse $\underline{38}$ Because of this, it was concluded 39 that this approach does not contain self-consistent checks of its accuracy, and hence could only be justified based on agreement with the experimental data and fundamental physical principles. In the next two sections we demonstrate, however, that theoretical approaches taking the screening effects into account are in disagreement with thermodynamics and available experimental data.

In the end of this section we discuss the claimed application region of the modified reflection coefficients taking into account screening effects. This is a familiar subject when describing conducting materials in an external electromagnetic field (we recall that Ref. 15 considers the fluctuating field in a similar way as an external one). In Ref. 15, the Boltzmann transport equation is applied in the nondegenerate continuum limit for sufficiently low density of charge carriers $n$ (intrinsic semiconductors). In this case the screening length is given by a specific Debye-Hückel expression,

$$
\frac{1}{\kappa}=\frac{1}{\kappa_{\mathrm{DH}}}=\sqrt{\frac{\varepsilon_{0} k_{B} T}{4 \pi e^{2} n}} .
$$


In fact this expression is applicable to charge carriers obeying Maxwell-Boltzmann statistics. It is obtained from the general representation for the screening length,, 32

$$
\frac{1}{\kappa}=\sqrt{\frac{\varepsilon_{0} D}{4 \pi \sigma(0)}},
$$

if one uses $\sigma(0)$ from Eq. (6) and Einstein's relation $18\left|32 D / \mu=k_{B} T /\right| e \mid$ valid in the case of Maxwell-Boltzmann statistics.

However, the application region of the modified reflection coefficients $r_{\mathrm{TM}, \mathrm{TE}}^{\bmod }$ with the Debye-Hückel screening length (14) is not restricted to only intrinsic semiconductors, but they are applicable to all materials where $n$ is not too large so that charge carriers are described by Maxwell-Boltzmann statistics. This means that it is legitimate to apply the coefficients $r_{\mathrm{TM}, \mathrm{TE}}^{\mathrm{mod}}$ to doped semiconductors with dopant concentration below critical, and to solids with ionic conductivity, etc.

Reference 15 claims that its approach "does not apply to metals, where the electron density is sufficiently large and the electron gas is degenerate". The Boltzmann transport equation, however, is equally applicable to classical and quantum systems. The only difference one should take into account is the type of statistics. In metals and metallic-type semiconductors charge carriers obey the quantum Fermi-Dirac statistics. Substituting Einstein's relation valid in the case of FermiDirac statistics, ${ }^{18 \mid 32} D / \mu=2 E_{F} /(3|e|)$, where $E_{F}=\hbar \omega_{p}$ is the Fermi energy, into Eq. (15), one arrives at the Thomas-Fermi screening length, 32

$$
\frac{1}{\kappa}=\frac{1}{\kappa_{\mathrm{TF}}}=\sqrt{\frac{\varepsilon_{0} E_{F}}{6 \pi e^{2} n}}
$$

With this definition of the parameter $\kappa$, it is justified to apply the modified reflection coefficients $r_{\mathrm{TM}, \mathrm{TE}}^{\mathrm{mod}}$ to metals. Thus, with the proper definition of the screening length, the suggested generalization of the Lifshitz theory, if at all meaningful, should be applicable to any material. The problem remains whether the screening effects are relevant to the Casimir force. This is discussed in the next sections.

\section{Inclusion of Screening Effects and Thermodynamics}

Here we perform the thermodynamic test of the Lifshitz formula (11) combined with the modified reflection coefficients $r_{\mathrm{TM}}^{\mathrm{mod}}$ from Eqs. (7), (8) and $r_{\mathrm{TE}}^{\mathrm{mod}}$. For this purpose the Casimir entropy at zero temperature will be calculated analytically. It is convenient to introduce the dimensionless variables $y=2 a q_{l}$ and $\zeta_{l}=\xi_{l} / \omega_{c} \equiv$ $2 a \xi_{l} / c$. Then the modified Casimir free energy takes the form

$$
\begin{gathered}
\mathcal{F}^{\mathrm{mod}}(a, T)=\frac{k_{B} T}{8 \pi a^{2}} \sum_{l=0}^{\infty} \int_{\zeta_{l}}^{\infty} y d y\left\{\ln \left[1-r_{\mathrm{TM}}^{\bmod ^{2}}\left(\mathrm{i} \zeta_{l}, y\right) \mathrm{e}^{-y}\right]\right. \\
\left.+\ln \left[1-r_{\mathrm{TE}}^{\bmod ^{2}}\left(\mathrm{i} \xi_{l}, y\right) \mathrm{e}^{-y}\right]\right\}
\end{gathered}
$$


In terms of dimensionless variables the reflection coefficient (7) can be rearranged as

$$
r_{\mathrm{TM}}^{\bmod }\left(\mathrm{i} \zeta_{l}, y\right)=\frac{\tilde{\varepsilon}_{l} y-\left[y^{2}+\left(\tilde{\varepsilon}_{l}-1\right) \zeta_{l}^{2}\right]^{1 / 2}-\left(y^{2}-\zeta_{l}^{2}\right)\left(\tilde{\varepsilon}_{l}-\varepsilon_{l}\right) \tilde{\eta}_{l}^{-1} \varepsilon_{l}^{-1}}{\tilde{\varepsilon}_{l} y+\left[y^{2}+\left(\tilde{\varepsilon}_{l}-1\right) \zeta_{l}^{2}\right]^{1 / 2}+\left(y^{2}-\zeta_{l}^{2}\right)\left(\tilde{\varepsilon}_{l}-\varepsilon_{l}\right) \tilde{\eta}_{l}^{-1} \varepsilon_{l}^{-1}},
$$

where

$$
\tilde{\eta}_{l}=2 a \eta_{l}=\left[y^{2}-\zeta_{l}^{2}+\kappa_{a}^{2} \frac{\varepsilon_{0} \tilde{\varepsilon}_{l}}{\varepsilon_{l}\left(\tilde{\varepsilon}_{l}-\varepsilon_{l}\right)}\right]^{1 / 2}, \quad \kappa_{a} \equiv 2 a \kappa .
$$

Note that all dielectric permittivities here are functions of $i \omega_{c} \zeta_{l}$. Below we do not use the explicit expression for the reflection coefficient $\tilde{r}_{\mathrm{TE}}\left(\mathrm{i} \zeta_{l}, y\right)$ because it coincides with the standard one, as defined in the Drude model approach, which was considered in detail in Ref. 4 .

Let us determine the behavior of the Casimir free energy (17) at low temperatures. We begin with the case of metals where $\kappa=\kappa_{\mathrm{TF}}$. For all metals the screening length is very small. As a result, at any reasonable separation distance between the plates, the dimensionless parameter $\kappa_{a}$ defined in (19) is very large and the inverse quantity $\beta_{a} \equiv 1 / \kappa_{a} \ll 1$ can be used as a small parameter. Expanding the reflection coefficient (18) up to the first power of the parameter $\beta_{a}$ one obtains

$$
\begin{aligned}
& r_{\mathrm{TM}}^{\mathrm{mod}}\left(\mathrm{i} \zeta_{l}, y\right)=\tilde{r}_{\mathrm{TM}}\left(\mathrm{i} \zeta_{l}, y\right)-2 \beta_{a} Z_{l}+O\left(\beta_{a}^{2}\right), \\
& Z_{l} \equiv \sqrt{\frac{\tilde{\varepsilon}_{l}\left(\tilde{\varepsilon}_{l}-\varepsilon_{l}\right)^{3}}{\varepsilon_{0} \varepsilon_{l}}} \frac{y\left(y^{2}-\zeta_{l}^{2}\right)}{\left[\tilde{\varepsilon}_{l} y+\sqrt{y^{2}+\left(\tilde{\varepsilon}_{l}-1\right) \zeta_{l}^{2}}\right]^{2}},
\end{aligned}
$$

where $\tilde{r}_{\mathrm{TM}}\left(\mathrm{i} \zeta_{l}, y\right)$ is the standard TM reflection coefficient calculated with the dielectric permittivity $\tilde{\varepsilon}\left(\mathrm{i} \omega_{c} \zeta_{l}\right)$. [It is given by Eq. (18) with the third terms in both the numerator and the denominator omitted.] From Eq. (20) one arrives at

$$
\ln \left[1-r_{\mathrm{TM}}^{\bmod }{ }^{2}\left(\mathrm{i} \zeta_{l}, y\right) \mathrm{e}^{-y}\right]=\ln \left[1-\tilde{r}_{\mathrm{TM}}^{2}\left(\mathrm{i} \zeta_{l}, y\right) \mathrm{e}^{-y}\right]+4 \beta_{a} \frac{\tilde{r}_{\mathrm{TM}}\left(\mathrm{i} \zeta_{l}, y\right) Z_{l}}{\mathrm{e}^{y}-\tilde{r}_{\mathrm{TM}}^{2}\left(\mathrm{i} \zeta_{l}, y\right)}+O\left(\beta_{a}^{2}\right) \text {. }
$$

Now we substitute (21) and the respective known expression for the TE contribution ${ }^{4}$ into (17). Calculating the sum with the help of the Abel-Plana formula, we obtain in perfect analogy to Ref. 4

$$
\begin{aligned}
\mathcal{F}^{\mathrm{mod}}(a, T) & =\mathcal{F}_{g p}(a, T)-\frac{k_{B} T}{16 \pi a^{2}} \int_{0}^{\infty} y d y \ln \left[1-r_{\mathrm{TE}, g p}^{2}(0, y) \mathrm{e}^{-y}\right] \\
& +\mathcal{F}^{(\gamma)}(a, T)+\beta_{a} \mathcal{F}^{(\beta)}(a, T),
\end{aligned}
$$

where $\mathcal{F}^{(\gamma)}(a, T)$ is determined by Eq. (17) in Ref. 4. It goes to zero together with its derivative with respect to temperature, when $T \rightarrow 0$. The quantity $\mathcal{F}^{(\beta)}(a, T)$ originates from the second contributions on the right-hand sides of (20), (21). Using the Abel-Plana formula,,$\sqrt[3]{ }$ it can be easily seen that $\mathcal{F}^{(\beta)}(a, T)=E^{(\beta)}(a)+O\left(T^{3} / T_{\text {eff }}^{3}\right)$ at low $T$. The Casimir free energy $\mathcal{F}_{g p}(a, T)$ is defined by substituting the dielectric permittivity of the generalized plasma-like model [Eq. (5) with $\gamma=0$ ] into the 
Lifshitz formula. It was found in Refs. 11, 12, and the respective thermal correction was shown to be of order $\left(T / T_{\text {eff }}\right)^{3}$ when $T \rightarrow 0$. The TE reflection coefficient calculated using the generalized plasma-like model at zero frequency entering (22) is given by

$$
r_{\mathrm{TE}, g p}(0, y)=\frac{c y-\sqrt{4 a^{2} \omega_{p}^{2}+c^{2} y^{2}}}{c y+\sqrt{4 a^{2} \omega_{p}^{2}+c^{2} y^{2}}} .
$$

Finally, calculating the Casimir entropy with inclusion of the screening effects,

$$
S^{\bmod }(a, T)=-\frac{\partial \mathcal{F}^{\bmod }(a, T)}{\partial T},
$$

using Eq. (22) in the limit $T \rightarrow 0$, one obtains

$$
S^{\bmod }(a, 0)=\frac{k_{B}}{16 \pi a^{2}} \int_{0}^{\infty} y d y \ln \left[1-r_{\mathrm{TE}, g p}^{2}(0, y) \mathrm{e}^{-y}\right]<0 .
$$

Thus, the Nernst heat theorem is violated and the theoretical approach leading to the modified reflection coefficients is thermodynamicly inconsistent. Note that this result is obtained for metals with perfect crystal lattices. In the presence of impurities the Casimir entropy abruptly jumps to zerd 21 at $T<10^{-3} \mathrm{~K}$. This, however, does not solve the problem of consistency with quantum statistical physics as discussed in Sec. 2.

Next we consider the low-temperature behavior of the Casimir free energy for dielectric materials which includes screening effects. This is also relevant for semiconductors with concentration of charge carriers below the critical value. For these materials $n$ is relatively small and $\kappa=\kappa_{\mathrm{DH}}$, as defined in Eq. (14), should be used. The derivation of the low-temperature behavior can be performed as in the case of two dielectric semispaces with the inclusion of dc conductivity $[5]$ For dielectric materials the small parameter $\beta_{l}$ is given by

$$
\beta_{l}=\frac{4 \pi \sigma(0)}{\xi_{l}} \quad(l \geq 1), \quad \sigma(0) \sim \exp \left(-\frac{C}{k_{B} T}\right),
$$

where $C$ is some constant having a different meaning for different classes of dielectrics. The parameter $\beta_{l}$ goes to zero when the temperature vanishes. Then we expand the modified reflection coefficients $r_{\mathrm{TM}, \mathrm{TE}}^{\bmod }\left(\mathrm{i} \zeta_{l}, y\right)$ with $l \geq 1$ in powers of the small parameter $\beta_{l}$,

$$
\begin{aligned}
& r_{\mathrm{TM}}^{\bmod }\left(\mathrm{i} \zeta_{l}, y\right)=r_{\mathrm{TM}}\left(\mathrm{i} \zeta_{l}, y\right)+\beta_{l} \frac{\varepsilon_{l} y\left[2 y^{2}+\left(\varepsilon_{l}-2\right) \zeta_{l}^{2}\right]}{\sqrt{y^{2}+\left(\varepsilon_{l}-1\right) \zeta_{l}^{2}}\left[\varepsilon_{l} y+\sqrt{y^{2}+\left(\varepsilon_{l}-1\right) \zeta_{l}^{2}}\right]^{2}}+O\left(\beta_{l}^{2}\right), \\
& r_{\mathrm{TE}}^{\bmod }\left(\mathrm{i} \zeta_{l}, y\right)=r_{\mathrm{TE}}\left(\mathrm{i} \zeta_{l}, y\right)+\beta_{l} \frac{y\left[y-\sqrt{y^{2}+\left(\varepsilon_{l}-1\right) \zeta_{l}^{2}}\right]}{\sqrt{y^{2}+\left(\varepsilon_{l}-1\right) \zeta_{l}^{2}}\left[y+\sqrt{y^{2}+\left(\varepsilon_{l}-1\right) \zeta_{l}^{2}}\right]}+O\left(\beta_{l}^{2}\right) .
\end{aligned}
$$

Here, the reflection coefficients $r_{\mathrm{TM}, \mathrm{TE}}$ are defined by Eq. (2) with the dielectric permittivity of core electrons (4). The Casimir free energy $\mathcal{F}(a, T)$ calculated with the coefficients $r_{\mathrm{TM}, \mathrm{TE}}$ vanishes with temperature as $\sim T^{3}$ (see Ref. 5 ). 
Now we substitute (27) in Eq. (17) and arrive at the following expression for the Casimir free energy taking the screening effects into account:

$\mathcal{F}^{\text {mod }}(a, T)=\mathcal{F}(a, T)+\frac{k_{B} T}{16 \pi a^{2}}\left\{\int_{0}^{\infty} y d y \ln \left[1-r_{\mathrm{TM}}^{\bmod }(0, y) \mathrm{e}^{-y}\right]+\operatorname{Li}_{3}\left(r_{0}^{2}\right)+Q(T)\right\}$,

where $\operatorname{Li}_{n}(z)$ is the polylogarithm function, $Q(T)$ vanishes exponentially $\sqrt{5}$ when $T \rightarrow 0, r_{0} \equiv\left(\varepsilon_{0}-1\right) /\left(\varepsilon_{0}+1\right)$ and

$$
r_{\mathrm{TM}}^{\bmod }(0, y)=\frac{\varepsilon_{0} \sqrt{y^{2}+\left(2 a \kappa_{\mathrm{DH}}\right)^{2}}-y}{\varepsilon_{0} \sqrt{y^{2}+\left(2 a \kappa_{\mathrm{DH}}\right)^{2}}+y}
$$

is the reflection coefficient (9) expressed in terms of the dimensionless variables. Calculating the negative derivative of both sides of (28) with respect to $T$, we obtain the asymptotic behavior of the Casimir entropy at low temperature

$$
\begin{aligned}
& S^{\bmod }(a, T)=S(a, T)-\frac{k_{B}}{16 \pi a^{2}}\left\{\int_{0}^{\infty} y d y \ln \left[1-r_{\mathrm{TM}}^{\bmod }(0, y) \mathrm{e}^{-y}\right]+\operatorname{Li}_{3}\left(r_{0}^{2}\right)\right. \\
& -8 a^{2} \varepsilon_{0} T \frac{\partial \kappa^{2}}{\partial T} \int_{0}^{\infty} d y \frac{y^{2} r_{\mathrm{TM}}^{\bmod }(0, y)}{\mathrm{e}^{y}-r_{\mathrm{TM}}^{\bmod ^{2}}(0, y)} \frac{1}{\sqrt{y^{2}+\left(2 a \kappa_{\mathrm{DH}}\right)^{2}}\left[\varepsilon_{0} \sqrt{y^{2}+\left(2 a \kappa_{\mathrm{DH}}\right)^{2}}+y\right]^{2}} \\
& \left.+Q(T)+T Q^{\prime}(T)\right\},
\end{aligned}
$$

where $S(a, T)$ is defined using $\mathcal{F}(a, T)$ and, thus, vanishes when $T \rightarrow 0$. It is easily seen that the last three terms in curly brackets on the right-hand side of this equation go to zero when $T$ goes to zero for any dielectric material.

The behavior of the first two terms in the curly brackets on the right-hand side of (30) when $T$ goes to zero is more involved. If $n(T)$ exponentially decays to zero with vanishing temperature (as is true for pure insulators and intrinsic semiconductors), then according to (14) so does $\kappa_{\mathrm{DH}}$. As a result, $r_{\mathrm{TM}}^{\bmod }(0, y) \rightarrow r_{0}$ and the first two terms in the curly brackets cancel. Then the Casimir entropy $S^{\bmod }(a, T)$ goes to zero when $T$ vanishes following the same law as $S(a, T)$, i.e., as $T^{2}$. This means that for insulators and intrinsic semiconductors the formalism under consideration is in agreement with the Nernst heat theorem.

However, there is a wide class of dielectric materials (such as doped semiconductors with dopant concentration below critical, dielectric like semimetals, certain amorphous semiconductors, and solids with ionic conductivity) for which $n$ does not go to zero when $T$ goes to zero. Although $\sigma(0)$ goes to zero exponentially fast for all dielectrics when $T$ goes to zero, for most of them this happens due to the vanishing mobility [see Eq. (6)]. For instance, the conductivity of $\mathrm{SiO}_{2}$ discussed in Ref. 14 is ionic in nature and is determined by the concentration of impuritues. For all such materials, in accordance with Eq. (14), $\kappa_{\mathrm{DH}} \rightarrow \infty$ when $T \rightarrow 0$. As a result, $r_{\mathrm{TM}}^{\bmod }(0, y) \rightarrow 1$ when $T$ goes to zero in accordance with (29). In this case we obtain 
from 30

$$
S^{\bmod }(a, 0)=\frac{k_{B}}{16 \pi a^{2}}\left[\zeta(3)-\operatorname{Li}_{3}\left(r_{0}^{2}\right)\right]>0
$$

i.e., the Casimir entropy is positive and depends on separation distance in violation of the Nernst heat theorem. This means that for a wide class of dielectric materials the proposed approach taking the screening effects into account is thermodynamically inconsistent 39 in the same way as the standard Lifshitz theory with the dc conductivity included.

We emphasize that the existence of dielectric materials for which $n$ does not go to zero but $\mu$ does go to zero when $T$ vanishes demonstrates that the reflection coefficient (18) at $\xi=0$ is ambiguous. In reality, for such materials $r_{\mathrm{TM}}^{\bmod }\left(0, k_{\perp}\right) \rightarrow 1$ when $T$ and $\mu$ simultaneously vanish. This is because $\kappa_{\mathrm{DH}} \rightarrow \infty$ when $T \rightarrow 0$ in disagreement with physical intuition that there should be no screening at zero mobility. This ambiguity is connected with the break of continuity of the reflection coefficient $r_{\mathrm{TM}}^{\bmod }\left(\mathrm{i} \xi, k_{\perp}\right)$ at the point $\xi=0, T=0$. If one takes the limit $T \rightarrow 0$ first, keeping $\xi=$ const $\neq 0$, the standard Fresnel reflection coefficients $r_{\mathrm{TM}}$ from Eq. (2) with no screening are reproduced. This property is preserved in the subsequent limiting transition $\xi \rightarrow 0$. Thus, as was already noted above, the violation of the Nernst heat theorem is caused by the break of continuity of the reflection coefficients at the point $(0,0)$ of the $(\xi, T)$-plane 34

Recently it was claimed 16 that the nonlocal approach leading to the reflection coefficient (9) with $\kappa=\kappa_{\mathrm{DH}}$ satisfies the Nernst theorem, specifically, for solids with ionic conductivity which is the conductivity of activation type. To prove this, Ref. 16 arbitrarily separates the thermal dependence of $\sigma(0)$ in Eqs (6), (26) from the mobility $\mu$ and attributes it to the "effective density of charges, which are able to move". This transfer of the temperature dependence from $\mu$ to $n$ is incorrect 39 because the commonly used density of charge carriers $n$ producing the effect of screening in ionic conductors is an independently measured quantity, which does not vanish with $T$. Independent measurements of all three quantities, conductivity, charge carrier concentration and mobility, demonstrate that "mobility has the dominating influence upon the conductivity-temperature dependence". 42 Nevertheless, Ref. 43 expresses doubts concerning the existence of dielectric materials whose density of charge carriers does not go to zero when $T$ vanishes, while the conductivity goes to zero due to vanishing mobility. It is common knowledge, however, that for semimetals of the dielectric type the Fermi energy is at a band where the density of states is not equal to zero. The number of charge carriers (electrons) near the Fermi surface in such dielectric materials is fixed because it is determined by the structure of the crystal lattice. Thus, the density of charge carriers is nonzero at any $T$ including $T=0 ! 44$ The same holds for certain of the amorphous semiconductors 45 and for doped semiconductors with dopant concentration below critical.

Recent Ref. 36 claims that it explicitly shows the satisfaction of the Nernst theorem in the Lifshitz theory with the modified reflection coefficients $r_{\mathrm{TM}, \mathrm{TE}}^{\mathrm{mod}}$ introduced 
in Ref. 15, According to Ref. 36, the Nernst theorem is satisfied "in systems with low density of carriers (intrinsic semiconductors, dielectrics, etc)". However, the presented proof uses an assumption that "the carrier density vanishes as $T \rightarrow 0$ " .36 Thus, wide classes of dielectric materials for which the density of charge carriers does not vanish when the temperature vanishes (doped semiconductors with dopant concentration below critical, semimetals of dielectric type, some amorphous semiconductors and solids with ionic conductivity) are simply excluded from consideration. As a result, the proof of Ref. 36 is in fact applicable to only insulators and intrinsic semiconductors, where the Fermi energy at $T=0$ lies in a band gap. For these materials the density of states at the Fermi energy is equal to zero. Thus, for insulators and intrinsic semiconductors the Nernst theorem is satisfied, as was proved above on the basis of Eq. (30). However, for the majority of dielectric materials $n$ does not go to zero with vanishing $T$ leading to the violation of the Nernst theorem in the modification of the Lifshitz theory proposed in Ref. 15 and also in Refs. 14, 16.

There is a remark in the literature 19 that the violation of Nernst's theorem for dielectric materials where $n$ does not go to zero with vanishing $T$ is "a pure misunderstanding" because "The materials under discussion are amorphous glass-like disordered bodies" for which "Nernst's theorem is not valid." This remark is erroneous in two aspects. First, as discussed above, there are dielectric materials with an ordered structure for which $n$ does not vanish with $T$ (dielectric like semimetals, for instance). Second, the violation of Nernst's theorem for disordered bodies is irrelevant to the Casimir entropy discussed here. As is correctly stated in Ref. 19, nonzero entropy of glass plates at $T=0$ is connected with the fact that they are simply not at an equilibrium state at low $T$. This entropy does not depend on separation between the plates and, thus, is not in contradiction with the Nernst theorem. By contrast, the Casimir entropy (31) is nonzero and depends on separation distance (i.e., on the volume of the system) for both ordered and disordered materials of plates (provided $n$ does not vanish with $T$ ) which is in contradiction with the Nernst theorem.

Thus, the substitution of the modified reflection coefficients into the Lifshitz formula leads to contradictions with thermodynamics. The physical reason for this is that the Lifshitz theory describes a system in thermal equilibrium whereas the modified reflection coefficients were obtained for a system that includes both drift and diffusion currents which are irreversible processes out of thermal equilibrium. As was emphasized at the beginning of this section, the Boltzmann transport equation describes irreversible processes which occur, for instance, in an external electric field. It is a far reaching extrapolation to apply this equation to the fluctuating electromagnetic field, as is done in Ref. [15. In an external electric field the system goes out of thermal equilibrium and the fluctuation-dissipation theorem is violated. This cannot happen and does not happen in the presence of fluctuating electromagnetic fields. One can conclude that the thermodynamic puzzles discussed above are artifacts of the application of the Lifshitz theory and Boltzmann transport equation 


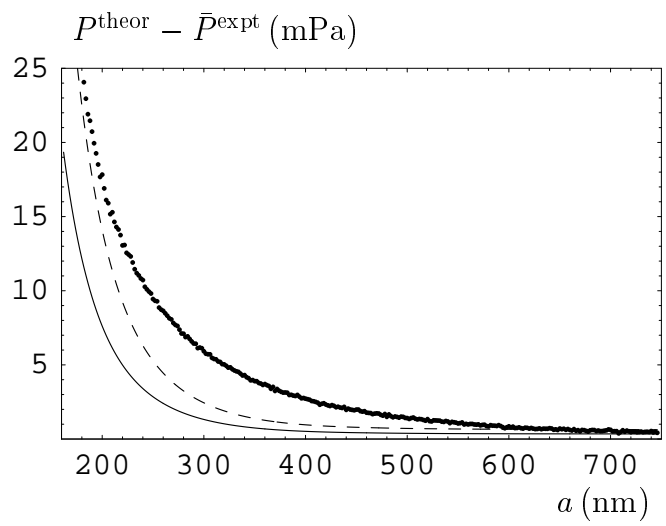

Fig. 3. The differences of the theoretical and the mean experimental Casimir pressures between the two Au plates versus separation are shown as dots. The theoretical results are calculated using the modified Lifshitz theory with the inclusion of screening effects. The solid and dashed lines indicate the boundaries of $95 \%$ and $99.9 \%$ confidence intervals, respectively.

outside of their range of applicability.

\section{Experimental Tests for the Influence of Screening Effects on the Casimir Force}

The theoretical predictions made by the approaches including screening effects in the Lifshitz theory can be compared with the recent experimental results from the measurement of the Casimir force between metal-metal and metal-semiconductor test bodies. In fact all three theoretical approaches $\frac{14-16}{-16}$ discussed above lead to almost coincident predictions. This is because the contributions from the zero frequency term in all these approaches are exactly the same and the contributions from all nonzero Matsubara frequencies are approximately equal.

We begin with the most precise experiment on the indirect measurement of the Casimir pressure between two gold plates by means of a micromechanical torsional oscillator ${ }^{7 / 8}$ In this experiment, the configuration of a sphere above a plate was used in the dynamic regime to determine the equivalent Casimir pressure in the configuration of two plates using the proximity force approximation. (This experiment was already mentioned in Sec. 2 in the comparison between the Drude model and the generalized plasma-like model in combination with the standard Lifshitz theory.) Note that Fig. 1(a) can be also used to compare the experimental data shown as crosses with the predictions of the theoretical approaches taking screening effects into account 14-16 The point is that at separations larger than $300 \mathrm{~nm}$ the approaches including the screening effects with $\kappa=\kappa_{\mathrm{TF}}$ lead to the same computational results for the Casimir pressure as the Drude model approach shown as the dark-gray band (the latter is plotted in the region from 500 to $600 \mathrm{~nm}$ ). As is seen in Fig. 1(a), the approaches taking into account screening effects are experimentally excluded at a 95\% confidence level. In Fig. 3 we use another method of 
comparison of these theoretical approaches with data over the entire measurement range from 162 to $750 \mathrm{~nm}$. Here, the differences between the theoretical and the mean experimental Casimir pressures are shown as dots. The solid and dashed lines indicate the boundaries of $95 \%$ and $99.9 \%$ confidence intervals, respectively. As is seen in the figure, the dots are outside the $95 \%$ confidence interval over the entire measurement range. At separations from 162 to $640 \mathrm{~nm}$ the theoretical approaches including the screening effects are experimentally excluded at the $99.9 \%$ confidence level. Recently it was claimed 43 that the electrostatic calibrations in the experiments of Refs. 17, 8] do not take into account "important systematic effects". This claim remains unjustified until some specific objection to the calibration procedures of Refs. 7, 8 described in more detail in Refs. 46, 47 is presented. It was claimed also $\frac{48}{4}$ that the comparison of experiment with theory, like in Figs. 1, 3, is irrelevant because the optical properties of the Au films used were not measured but taken from tables. However, computations performed in Ref. 49 demonstrated that the use of any alternative set of optical data only increases disagreement between the Drude model approach or approaches of Refs. 14 16 and the experimental data.

Next we compare the theoretical predictions with the inclusion of the screening effects with the measurement data of the experiment on the modulation of the Casimir force with light 27/28 (This experiment was also mentioned in Sec. 2.) Here, the difference of the Casimir forces $F_{\text {diff }}$ between an Au coated sphere and a Si plate was measured in the presence, and in the absence, of laser light on the plate. In the absence of light, the concentration of charge carriers in Si was much below the critical value, i.e., $\mathrm{Si}$ was in a dielectric state. In the presence of light, the concentration of charge carriers in Si was above the critical value, i.e., Si was in a metallic state (all values of respective parameters are listed in Ref. 28).

The theoretical predictions with the inclusion of screening effects are numerically almost the same, as in the standard Lifshitz theory, for the case when the Si plate is in the bright phase, but differ measurably when the Si plate is in the dark phase in comparison with the calculation where the conductivity of $\mathrm{Si}$ in the dark phase is simply neglected (see Sec. 2). It is not possible, however, to conclusively compare experiment with theory taking the screening effects into account at a $95 \%$ confidence level. Below we compare the experimental data whose total experimental errors are determined at a $70 \%$ confidence level with the theoretical bands computed with inclusion of screening effects. Note that in the dark phase $\kappa=\kappa_{\mathrm{DH}}$ was used in the computations. In the bright phase the computational results for the Casimir force are almost the same for both $\kappa=\kappa_{\mathrm{DH}}$ and $\kappa=\kappa_{\mathrm{TF}}$. The width of the bands is found at the same $70 \%$ confidence level as the experimental errors. This width is mostly determined by the error in the charge carrier densities used in the computations. In Fig. 4(a,b) the experimental difference Casimir forces, $F_{\text {diff }}^{\text {expt }}$ (the force in the bright phase minus the force in the dark phase) are shown as crosses for different measurements with absorbed power $P_{w}=9.3 \mathrm{~mW}$ and $8.5 \mathrm{~mW}$, respectively. The theoretical bands for $F_{\text {diff }}^{\text {theor }}$ computed with the inclusion of the screening effects lie in between the dashed lines. As is seen in Fig. 4(a,b), the theoretical approach taking 

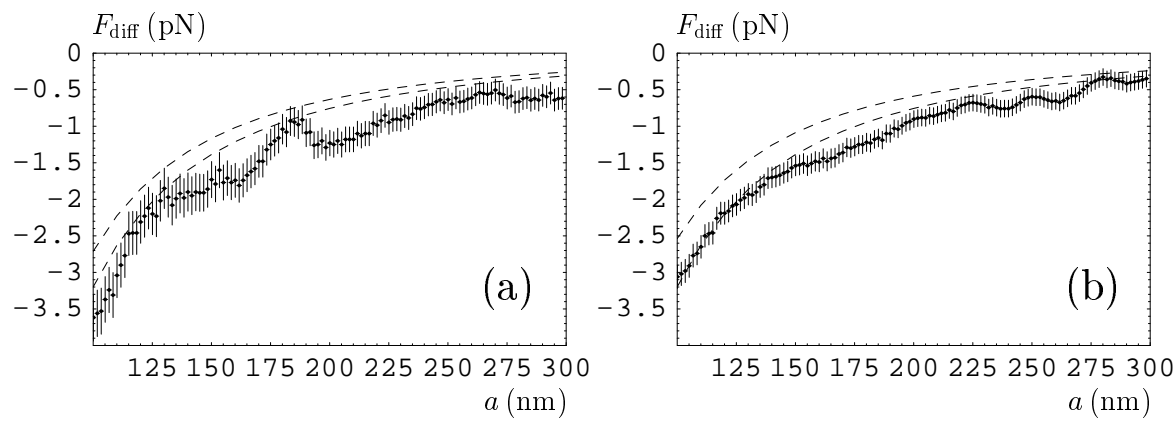

Fig. 4. (a) The measured force differences are shown as crosses versus separation $a$ for the absorbed power (a) $P_{w}=9.3 \mathrm{~mW}$ and (b) $P_{w}=8.5 \mathrm{~mW}$. The theoretical results calculated with inclusion of screening effects lie between the dashed lines.

into account the screening effects is excluded by the data at almost all separation distances within the measurement range from 100 to $300 \mathrm{~nm}$.

In Fig. 5 the experimental data from one more repetition of the same experiment with a lower absorbed power $\left(P_{w}=4.7 \mathrm{~mW}\right)$ are compared with the theoretical approaches taking the screening effects into account. In Fig. 5(a) the experimental data are shown as crosses. The theoretical results computed with the inclusion of the screening effects belong to the band in between the two dashed lines. As is seen in Fig. 5(a), below $200 \mathrm{~nm}$ and above $275 \mathrm{~nm}$ the theory is inconsistent with data. In Fig. 5(b) the same data are compared with the same theoretical approach using another method of comparison. Here, theoretical minus experimental differences of the Casimir forces are plotted as dots. The solid line shows the upper boundary of the $70 \%$ confidence intervals taking into account all experimental and theoretical errors. It is seen that below $185 \mathrm{~nm}$ and above $280 \mathrm{~nm}$ all dots are outside the confidence intervals. As a result, Figs. $4(\mathrm{a}, \mathrm{b})$ and $5(\mathrm{a}, \mathrm{b})$ conclusively indicate that the theoretical approaches taking into account screening effects are excluded by the
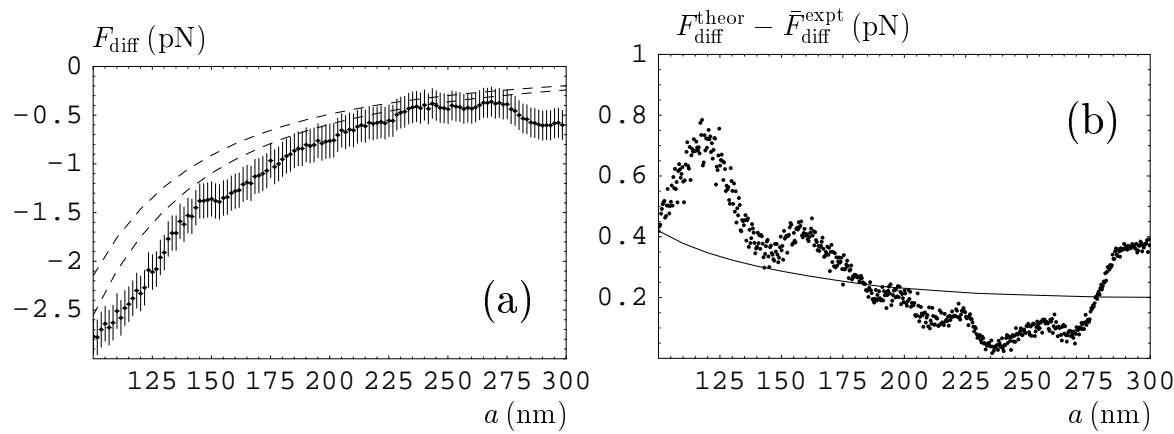

Fig. 5. (a) The measured force differences are shown as crosses versus separation $a$ for the absorbed power $P_{w}=4.7 \mathrm{~mW}$. The theoretical results calculated with inclusion of screening effects lie between the dashed lines. (b) Theoretical minus mean experimental differences of the Casimir force for the Lifshitz theory with inclusion of screening effects. The solid line indicates $70 \%$ confidence intervals. 
data of the optical modulation experiment $27 / 28$ at a $70 \%$ confidence level.

In contrast to this conclusion, it was claimed 16 that "the experiment can hardly distinguish between nonlocal theory and local theory with zero conductivity of Si." (We recall that according to Sec. 2, Fig. 2, the local theory with dc conductivity of Si neglected in the dark phase is in perfect agreement with the data.) The comparison between the theoretical and experimental results in Ref. [16 is, however, irregular. 39 In Fig. 1(a) of Ref. 16 the experimental data [taken from Fig. 1(a) of Ref. 29 with no indication of the source] are shown with errors determined at a $70 \%$ confidence level. In the same figure the theoretical band for the nonlocal approach including the screening effects is obtained from the uncertainty in $n, \Delta n=0.4 \times 10^{19} \mathrm{~cm}^{-3}$, determined 28 at a $95 \%$ confidence level. The reader of Ref. 16 is not informed about the confidence levels used. This comparison of experiment with theory is thus confusing. In our Fig. 4(a) the same data are compared with the predictions of the nonlocal approach (the band between the dashed lines), where the band width is determined at the same $70 \%$ confidence level, i.e., with $\Delta n=0.3 \times 10^{19} \mathrm{~cm}^{-3}$, as the errors of the data. It can be clearly seen that the nonlocal approach is excluded by the data at a $70 \%$ confidence level.

Regarding Fig. 1(b) in Ref. 16, it shows both the data and the theoretical bands at a $95 \%$ confidence level and restricts the region of separations only from 100 to $150 \mathrm{~nm}$. However, 29 these data cannot be conclusively compared with the nonlocal approach at such a high confidence. The correct comparison between the data and the nonlocal approach for this measurement set of the optical modulation experiment over the entire separation range is presented in our Fig. 4(b). It can be clearly seen that data are inconsistent with the nonlocal approach including the screening effects over a wide range of separations.

\section{Conclusions and Discussion}

In the above, we have reviewed problems with the Lifshitz theory when the drift current of conduction electrons in metals, or the dc conductivity at $T \neq 0$ in dielectrics, are included in the model of the dielectric response. It was shown that these phenomena violate thermal equilibrium which is the basic applicability condition of the Lifshitz theory. Because of this, the inclusion of the drift current into the Lifshitz formula results in thermodynamic and experimental inconsistencies.

The attempts to avoid these problems introduce modified reflection coefficients taking into account screening effects and diffusion currents 14 -16 we show (see also Refs. 39 41) that the Lifshitz formula combined with the modified reflection coefficients leads to nonzero Casimir entropy at $T=0$ depending on the separation distance between the plates. Thus, the proposed approaches violate the third law of thermodynamics. These approaches are also demonstrated to be inconsistent with the measurement data of two experiments. For metal-metal test bodies they are excluded experimentally at a $99.9 \%$ confidence level. In the configuration of metalsemiconductor test bodies the exclusion is confirmed at a $70 \%$ confidence level. 
The reason for the failure of the theoretical approaches, including the screening effects and diffusion currents into the Lifshitz theory, is that these processes violate thermal equilibrium which is the basic applicability condition of the Lifshitz theory. Both the drift and diffusion currents are irreversible phenomena which take place out of thermal equilibrium. By contrast, the dispersion forces are physical phenomena of fluctuating nature which go on in thermal equilibrium. One arrives at the conclusion that there is a deep difference between external and fluctuating electromagnetic fields which might not be sufficiently reflected in the mathematical formalism of quantum theory. Future studies will show how important this conclusion is for wider ranges of physical phenomena beyond the scope of dispersion forces.

\section{Acknowledgments}

R.S.D. acknowledges NSF support through Grants No. CCF-0508239 and PHY0701636, and from the Nanoscale Imaging Center at IUPUI. E.F. was supported in part by DOE under Grant No. DE-76ER071428. U.M., G.L.K. and V.M.M. were supported by the NSF Grant No. PHY0653657 (computations of the Casimir force) and DOE Grant No. DE-FG02-04ER46131 (measurement of the Casimir force differences). G.L.K. and V.M.M. were also partially supported by Deutsche Forschungsgemeinschaft, Grant No. 436 RUS 113/789/0-4.

\section{References}

1. H. B. G. Casimir, Proc. K. Ned. Akad. Wet. 51793 (1948).

2. E. M. Lifshitz, Sov. Phys. JETP 2, 73 (1956).

3. M. Bordag, U. Mohideen and V. M. Mostepanenko, Phys. Rep. 353, 1 (2001).

4. V. B. Bezerra, G. L. Klimchitskaya, V. M. Mostepanenko and C. Romero, Phys. Rev. A 69, 022119 (2004).

5. B. Geyer, G. L. Klimchitskaya and V. M. Mostepanenko, Phys. Rev. D 72, 085009 (2005).

6. R. S. Decca, D. López, E. Fischbach, G. L. Klimchitskaya, D. E. Krause and V. M. Mostepanenko, Ann. Phys. (N.Y.) 318, 37 (2005).

7. R. S. Decca, D. López, E. Fischbach, G. L. Klimchitskaya, D. E. Krause and V. M. Mostepanenko, Phys. Rev. D 75, 077101 (2007).

8. R. S. Decca, D. López, E. Fischbach, G. L. Klimchitskaya, D. E. Krause and V. M. Mostepanenko, Eur. Phys. J. C 51, 963 (2007).

9. G. L. Klimchitskaya and V. M. Mostepanenko, J. Phys. A: Math. Theor. 41, 312002 (2008).

10. G. L. Klimchitskaya, U. Mohideen and V. M. Mostepanenko, J. Phys. A: Math. Theor. 40, F841 (2007).

11. B. Geyer, G. L. Klimchitskaya and V. M. Mostepanenko, J. Phys. A: Math. Theor. 40, 13485 (2007).

12. V. M. Mostepanenko and B. Geyer, J. Phys. A: Math. Theor. 41, 164014 (2008).

13. G. L. Klimchitskaya and B. Geyer, J. Phys. A: Math. Theor. 41, 164032 (2008).

14. L. P. Pitaevskii, Phys. Rev. Lett. 101, 163202 (2008).

15. D. A. R. Dalvit and S. K. Lamoreaux, Phys. Rev. Lett. 101, 163203 (2008).

16. V. B. Svetovoy, Phys. Rev. Lett. 101, 163603 (2008). 
17. V. A. Parsegian, Van der Waals Forces: A Handbook for Biologists, Chemists, Engineers, and Physicists (Cambridge University Press, Cambridge, 2005).

18. N. W. Ashcroft and N. D. Mermin, Solid State Physics (Saunders College, Philadelphia, 1976).

19. L. P. Pitaevskii, arXiv:0811.3081; Phys. Rev. Lett., to appear.

20. I. Brevik, J. B. Aarseth, J. S. Høye and K. A. Milton, Phys. Rev. E 71, 056101 (2005).

21. J. S. Høye, I. Brevik, S. A. Ellingsen and J. B. Aarseth, Phys. Rev. E 75, 051127 (2007).

22. V. B. Bezerra, R. S. Decca, E. Fischbach, B. Geyer, G. L. Klimchitskaya, D. E. Krause, D. López, V. M. Mostepanenko and C. Romero, Phys. Rev. E 73, 028101 (2006).

23. G. L. Klimchitskaya and V. M. Mostepanenko, Phys. Rev. E 77, 023101 (2008).

24. R. S. Decca, E. Fischbach, G. L. Klimchitskaya, D. E. Krause, D. López and V. M. Mostepanenko, Phys. Rev. D 68, 116003 (2003).

25. B. Geyer, G. L. Klimchitskaya and V. M. Mostepanenko, Int. J. Mod. Phys. A 21, 5007 (2006).

26. B. Geyer, G. L. Klimchitskaya and V. M. Mostepanenko, Ann. Phys. (N. Y.) 323, 291 (2008).

27. F. Chen, G. L. Klimchitskaya, V. M. Mostepanenko and U. Mohideen, Optics Express 15, 4823 (2007).

28. F. Chen, G. L. Klimchitskaya, V. M. Mostepanenko and U. Mohideen, Phys. Rev. B 76, 035338 (2007).

29. G. L. Klimchitskaya, U. Mohideen and V. M. Mostepanenko, J. Phys. A: Math. Theor. 41, 432001 (2008).

30. V. V. Bryksin and P. M. Petrov, Phys. Solid State 50, 229 (2008).

31. D. Kondepugi and I. Prigogine, Modern Thermodynamics (John Wiley \& Sons, New York, 1998).

32. J.-N. Chazalviel, Coulomb Screening of Mobile Charges: Applications to Material Science, Chemistry and Biology (Birkhauser, Boston, 1999).

33. K. S. Førland, T. Førland and S. K. Ratkje, Irreversible Thermodynamics (John Wiley \& Sons, New York, 1988).

34. F. Intravaia and C. Henkel, J. Phys. A: Math. Theor. 41, 164018 (2008).

35. G. L. Klimchitskaya and V. M. Mostepanenko, Phys. Rev. B 75, 036101 (2006).

36. D. A. R. Dalvit and S. K. Lamoreaux, arXiv:0812.0557 J. Phys.: Conf. Ser. 161, 012009 (2009).

37. K. L. Kliewer and R. Fuchs, Phys. Rev. 172, 607 (1968).

38. J. T. Foley and A. J. Devaney, Phys. Rev. B 12, 3104 (1975).

39. G. L. Klimchitskaya, U. Mohideen and V. M. Mostepanenko, arXiv:0810.3247.

40. R. S. Decca, E. Fischbach, B. Geyer, G. L. Klimchitskaya, D. E. Krause, D. López, U. Mohideen and V. M. Mostepanenko, arXiv:0810.3244 Phys. Rev. Lett., to appear.

41. B. Geyer, G. L. Klimchitskaya, U. Mohideen and V. M. Mostepanenko, arXiv:0810.3243, Phys. Rev. Lett., to appear.

42. H. J. Schütt and E. Gerdes, J. Non-Cryst. Sol. 144, 14 (1992).

43. D. A. R. Dalvit and S. K. Lamoreaux, arXiv:0812.1056 Phys. Rev. Lett., to appear.

44. N. F. Mott, Metal-Insulator Transitions (Taylor and Francis, London, 1990).

45. P. Nagels, in: Amorphous Semiconductors, ed. M. H. Brodsky (Springer, Berlin, 1979).

46. R. S. Decca, E. Fischbach, B. Geyer, G. L. Klimchitskaya, D. E. Krause, D. López, U. Mohideen and V. M. Mostepanenko, arXiv:0809.3576, Phys. Rev. A 79, 026101 (2009).

47. R. S. Decca and D. López, Int. J. Mod. Phys. A 24, 1748 (2009).

48. V. B. Svetovoy, arXiv:0811.3423. 
January 8, 2019 18:39 WSPC/INSTRUCTION FILE mostepFS7

22 V. M. Mostepanenko et al.

49. V. M. Mostepanenko, J. Phys.: Conf. Ser. 161, 012003 (2009). 